\title{
Baja incidencia de cardiopatía clínicamente significativa en niños en edad escolar tras padecer COVID-19
}

The low incidence of clinically significant heart disease in school-age children following COVID-19

\author{
Adam W. Powell $^{a} \mathbb{D}$, Christopher J. Statile $\mathbb{D}^{\mathbb{D}}$, Nicolas L. Madsen ${ }^{a}$, Allison A. Divanovic ${ }^{a}$, Sean M. Lang $\mathbb{D}^{\mathbb{D}}$
}

\section{RESUMEN}

No es clara la carga de morbimortalidad de la lesión cardíaca clínicamente evidente secundaria a la enfermedad por coronavirus de 2019 (COVID-19) en los niños en edad escolar. A lo largo de 12 meses, en un importante hospital pediátrico académico en la región del medio oeste de Estados Unidos, hubo 1481 casos de COVID-19 sin hospitalización en niños en edad escolar por lo demás sanos, en quienes se hicieron 195 pruebas cardíacas. Si bien aparecieron hallazgos fortuitos, no se descubrió ninguna patología cardíaca relacionada con la COVID-19. Además, 3\% de los niños solamente tuvieron síntomas cardíacos agudos que requirieron una evaluación por el área de cardiología pediátrica. Los niños que no fueron hospitalizados por COVID-19 tienen un riesgo muy bajo de desarrollar daño cardíaco clínicamente significativo y son más propensos a presentar hallazgos fortuitos.

Palabras clave: COVID-19, cardiopatías, secuelas, COVID-19 persistente.

http: / / dx.doi.org/10.5546/ aap.2022.54

Texto completo en inglés:

http:/ / dx.doi.org/10.5546/ aap.2022.eng.54

Cómo citar: Powell AW, Statile CJ, Madsen NL, Divanovic AA, Lang SM. Baja incidencia de cardiopatía clínicamente significativa en niños en edad escolar tras padecer COVID-19. Arch Argent Pediatr 2022;120(1):54-57. a. Departamento de Pediatría, Facultad de Medicina de la University of Cincinnati, Cincinnati, Ohio, Estados Unidos. The Heart Institute, Cincinnati Children's Hospital Medical Center, Cincinnati, Ohio, Estados Unidos.

\section{Correspondencia:}

Adam W. Powell: Adam.Powell@cchmc.org

Financiamiento: Ninguno.

Conflicto de intereses: Ninguno que declarar.

Recibido: 13-7-2021

Aceptado: 2-9-2021

\section{INTRODUCCIÓN}

La lesión cardíaca clínicamente significativa es una complicación temida en los niños que padecen la enfermedad por coronavirus de 2019 (COVID-19), lo que provoca cancelaciones de actividades deportivas y el desarrollo de múltiples pautas de "regreso a las actividades". ${ }^{1}$ Si bien la prevalencia de lesión cardíaca clínicamente significativa tras padecer COVID-19 (definida como miocarditis, inflamación o disfunción del miocardio) parece ser baja en los atletas universitarios competitivos que no requieren hospitalización, no se ha estudiado adecuadamente el daño cardíaco clínicamente significativo en niños en edad escolar no hospitalizados. ${ }^{2,3}$ El objetivo de este estudio fue establecer las características adicionales de la lesión cardíaca clínicamente significativa observada en los niños en edad escolar no hospitalizados que padecieron COVID-19.

\section{MÉTODOS}

Se hizo una revisión retrospectiva de las historias clínicas de todos los pacientes en edad escolar (5 a 18 años) no hospitalizados que tuvieron una prueba positiva en COVID-19 hecha en un importante hospital pediátrico académico en la región del medio oeste de Estados Unidos o evaluados por el área de cardiología pediátrica debido a un estudio positivo en COVID-19 en un establecimiento externo, entre el 1 de marzo de 2020 y el 28 de febrero de 2021. Para limitar la participación en el estudio a pacientes con manifestaciones de COVID-19 aguda y evitar a los pacientes con síntomas de COVID-19 persistente, se excluyó a los pacientes evaluados $>2$ meses después de la prueba positiva. Se excluyó a los niños con comorbilidades crónicas y síndrome inflamatorio multisistémico pediátrico (MIS-C, por su sigla en inglés). Se registraron los resultados de las pruebas cardíacas.

El consejo de revisión institucional del hospital aprobó el estudio. 


\section{RESULTADOS}

En total, se incluyeron en el estudio 1481 pacientes con COVID-19 en edad escolar por lo demás sanos (1413 con prueba positiva en el hospital y 69, fuera del hospital) (Figura 1). No hubo muertes en esta cohorte. De los 117 pacientes que consultaron con cardiología, el $44 \%$ (52/117) fueron derivados por síntomas. El $13 \%(195 / 1481)$ de la cohorte de pacientes con COVID-19 en edad escolar no hospitalizados se sometieron a pruebas cardíacas, entre otras, electrocardiograma, ecocardiograma, concentración sérica de troponina y resonancia magnética cardíaca (RMC). Los resultados de las pruebas cardíacas se muestran en la Tabla 1. No hubo casos de patología decisiva relacionada con la COVID-19 (miocarditis, inflamación o disfunción del miocardio).
Los hallazgos notables se muestran en la Figura 1. Cabe destacar que un paciente presentó un hallazgo preocupante en el ecocardiograma inicial de dilatación de la arteria coronaria izquierda, pero los tamaños cardíacos observados en la tomografía computada (TC) posterior fueron normales. Se consideró que el ecocardiograma inicial había sido un resultado positivo falso ya que la TC del corazón es más específica para medir las arterias coronarias. Este paciente no recibió ningún tratamiento (p. ej., inmunoglobulina intravenosa, aspirina) y recibió el alta de cardiología cuando se demostró que las arterias coronarias eran normales.

\section{DISCUSIÓN}

Entre todos los niños con COVID-19 no hospitalizados, no se encontró ningún caso

TABLA 1. Evaluación cardiaca para toda la cohorte

\begin{tabular}{|c|c|}
\hline Total de pruebas positivas & 1481 \\
\hline Sexo & Masculino 763, Femenino 718 \\
\hline Edad (años), media \pm desviación estándar & $13,8 \pm 3,4$ \\
\hline Total de consultas con cardiología & 117 \\
\hline Total de ECG & 191 \\
\hline Ritmo predominante & 186 Sinusal \\
\hline & 5 Auricular ectópico \\
\hline Hallazgos en el ECG & $\begin{array}{l}158 \text { Sin hallazgos patológicos } \\
8 \text { Prolongación del intervalo QT } \\
4 \text { Cambios inespecíficos en onda T/segmento ST } \\
3 \text { Eje desviado a la izquierda } \\
2 \text { Contracciones ventriculares prematuras } \\
2 \text { Preexcitación ventricular } \\
2 \text { Dilatación auricular izquierda } \\
1 \text { Dilatación auricular derecha } \\
7 \text { Hipertrofia ventricular izquierda } \\
1 \text { Hipertrofia ventricular derecha } \\
1 \text { Bloqueo de la rama derecha del haz de His } \\
1 \text { Ondas Q profundas en derivaciones inferiores / laterales } \\
1 \text { Contracciones auriculares prematuras }\end{array}$ \\
\hline $\begin{array}{l}\text { Total de ecocardiogramas } \\
\text { Hallazgos }\end{array}$ & $\begin{array}{l}32 \\
23 \text { Normales } \\
2 \text { Válvula aórtica bicúspide } \\
1 \text { Dilatación moderada de la aorta ascendente/ raíz aórtica } \\
1 \text { Dilatación leve de la arteria coronaria derecha } \\
1 \text { Dilatación leve de la arteria coronaria izquierda } \\
1 \text { Hipertrofia ventricular izquierda leve } \\
1 \text { Trabeculaciones leves en el ventrículo izquierdo } \\
1 \text { Agujero oval persistente } \\
1 \text { Conducto arterial persistente insignificante }\end{array}$ \\
\hline Total de RM/TC cardíaca & 4 \\
\hline Total de pruebas de esfuerzo & 2 \\
\hline Total de extracciones de troponina T sérica & 12 \\
\hline Media \pm desviación estándar (ng/1) & $2,0 \pm 2,5($ normal $<15 \mathrm{ng} / \mathrm{l})$ \\
\hline
\end{tabular}


de patología cardíaca decisiva relacionada con la COVID-19. En cambio, la mayoría de las patologías observadas correspondieron a hallazgos fortuitos (válvula aórtica bicúspide, contracciones ventriculares prematuras) o a resultados positivos falsos ("anomalías" de la arteria coronaria según el ecocardiograma con una TC cardíaca posterior normal). Además, pocos niños en edad escolar con COVID-19 $(\sim 3 \%)$ tienen síntomas agudos que finalmente requieran una evaluación con cardiología. Los patrones de derivación al área de cardiología resultan mayormente del cumplimiento de las pautas de regreso a las actividades, incluida la preocupación por anomalías detectadas en el ECG. ${ }^{4}$

Moulson y col., llevaron a cabo un estudio de cohortes observacional, prospectivo y de gran tamaño con 42 universidades y más de

Figura 1. Diagrama de flujo de pacientes con COVID-19 de entre 5 y 18 años de edad y distribución de pruebas y evaluaciones cardíacas en esta cohorte. Los hallazgos notables de la evaluación cardíaca se muestran debajo

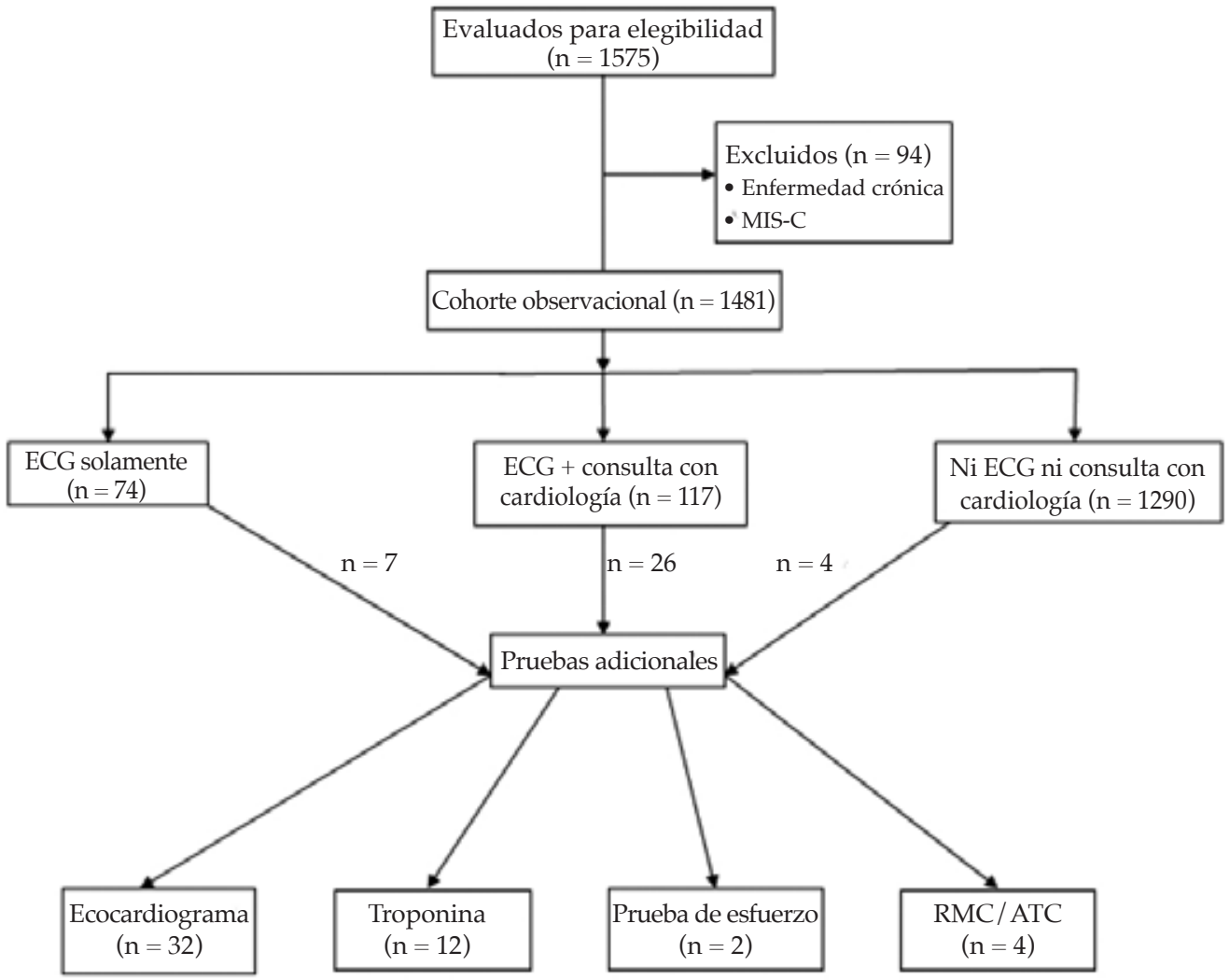

Ejemplos de pacientes:

1. Mujer de 15 años, arteria coronaria izquierda levemente dilatada según el ecocardiograma, posteriormente normal según la TC del corazón 2 semanas después, sin seguimiento.

2. Hombre de 18 años, preocupación por origen anómalo de arteria coronaria derecha según el ecocardiograma, normal según la TC del corazón, sin seguimiento.

3. Hombre de 14 años, ondas T anómalas en las derivaciones inferiores y laterales del ECG, normalizadas en la prueba de esfuerzo, hipertrofia ventricular izquierda leve según el ecocardiograma y la RMC, seguimiento programado para control de la hipertrofia ventricular izquierda leve.

4. Hombre de 17 años, dilatación leve de ventrículos izquierdo y derecho con trabeculaciones prominentes en el ventrículo izquierdo según la RMC, seguimiento programado para control de las trabeculaciones en el ventrículo izquierdo.

5. Hombre de 16 años, contracciones ventriculares prematuras frecuentes según el ECG, ecocardiograma normal, supresión de extrasístole ventricular durante la prueba de esfuerzo, se consideró no relacionada con la COVID-19, seguimiento en 6 meses.

6. Dos pacientes con válvula aórtica bicúspide, un paciente con conducto arterial persistente insignificante, hallazgos fortuitos.

Abreviaturas: MIS-C: síndrome inflamatorio multisistémico pediátrico (por su sigla en inglés), ECG: electrocardiograma, RMC: resonancia magnética cardíaca, ATC: angiografía por tomografía computada. 
3000 atletas universitarios sometidos a una evaluación cardíaca. Ese estudio halló que $<1 \%$ de los atletas con COVID-19 tuvieron afectación cardíaca sin eventos cardíacos adversos relacionados con la COVID-19. ${ }^{2}$ En nuestra cohorte, de menor edad, el hallazgo similar de ausencia de eventos cardíacos inducidos por la COVID-19 no es sorprendente ya que los pacientes pediátricos más jóvenes presentan tasas inferiores de síntomas agudos significativos. ${ }^{5} \mathrm{Sin}$ embargo, esto no ha detenido la preocupación de los profesionales pediátricos y las familias acerca de la seguridad de retomar la actividad física. Es posible que algunas pautas de regreso a las actividades actuales sean excesivamente conservadoras, independientemente de la gravedad de la enfermedad. ${ }^{1}$ De hecho, como demuestran nuestros resultados, estas pruebas cardíacas diagnósticas permiten que se hagan hallazgos fortuitos en estos pacientes en lugar de relacionados con la COVID-19, lo que podría llevar a un aumento de la sobreexplotación de recursos.

La baja prevalencia de lesión cardíaca en este y otros estudios implica que las preocupaciones sobre el daño cardíaco no deben orientar las decisiones sobre si un niño debe retomar o no la educación presencial o las actividades deportivas. Estas decisiones deben estar impulsadas por consideraciones de salud pública y la reducción de la transmisión de la enfermedad. La baja probabilidad de afectación cardíaca relacionada con la COVID-19 sugiere que un enfoque en la prevención secundaria sería más eficaz, como garantizar que los entrenadores y el personal de la escuela estén capacitados en RCP y el uso de un desfibrilador externo automático. ${ }^{6}$

Este estudio tuvo algunas limitaciones importantes. En primer lugar, este fue un estudio unicéntrico retrospectivo y tal vez no refleje adecuadamente la carga de morbimortalidad de la COVID-19 en otras áreas del mundo. En segundo lugar, ya que este estudio se centró exclusivamente en la enfermedad clínica, no se evaluó la presencia de disfunción cardíaca subclínica, aunque la significancia de la cardiopatía subclínica es motivo de intensos debates. ${ }^{2,7}$ Por último, si bien afortunadamente nuestra cohorte no demostró anomalías coronarias agudas, este estudio no buscaba determinar el riesgo de la afectación de la arteria coronaria a largo plazo en los niños con COVID-19 leve. Si bien la evidencia sobre la afectación de la arteria coronaria se centró mayormente en los niños con COVID-19 grave o MIS-C, se requieren estudios adicionales en pacientes pediátricos con infección menos grave para determinar si necesitan vigilancia cardíaca continua. ${ }^{8}$

A modo de conclusión, los niños que no fueron hospitalizados por COVID-19 tienen un riesgo muy bajo de desarrollar daño cardíaco clínicamente significativo y son más propensos a presentar hallazgos fortuitos. Esperamos que nuestra experiencia aporte un contexto sobre la necesidad de hacer pruebas cardíacas en los niños en edad escolar asintomáticos que no requieren hospitalización.

\section{REFERENCIAS}

1. American Academy of Pediatrics. COVID-19 interim guidance: return to sports and physical activity. Updated March 1,2021. Accessed May 13, 2021. Availablein: https: / / services.aap.org/en/pages / 2019-novel-coronaviruscovid-19-infections / clinical-guidance/ covid-19-interimguidance-return-to-sports /

2. Moulson N, Petek BJ, Drezner JA, Harmon KG, et al. SARSCoV-2Cardiac Involvement in Young Competitive Athletes. Circulation. 2021; 144(4):256-66.

3. Dyal J, Schwartz AD, Oster M, Mahle W. Cardiac screening after COVID-19 infection in children: is electrocardiogram warranted? J Am Coll Cardiol. 2021; 77(18):3184.

4. Zimmermann $\mathrm{P}$, Curtis N. Coronavirus Infections in Children Including COVID-19: An Overview of the Epidemiology, Clinical Features, Diagnosis, Treatment and Prevention Options in Children. Pediatr Infect Dis J. 2020; 39(5):355-68.

5. Gartenberg AJ, White TJ, Dang K, Shah M, et al. Assessing the Utility of Screening Electrocardiograms in Pediatric Patients Following COVID-19. Cardiol Young. 2021.Págs.1-7.

6. DreznerJA, ToresdahlBG, Rao AL, HusztiE, etal. Outcomes from sudden cardiac arrest in US high schools: a 2-year prospective study from the National Registry for AED Use in Sports. Br J Sports Med. 2013; 47(18):1179-83.

7. Brito D, Meester S, Yanamala N, Patel H, et al. High Prevalence of Pericardial Involvement in College Student Athletes Recovering From COVID-19. JACC Cardiovasc Imaging. 2021; 14(13):541-55.

8. Feldstein LR, Tenforde MW, Friedman KG, Newhams M, et al. Overcoming COVID-19 Investigators. Characteristics and Outcomes of US Children and Adolescents With Multisystem Inflammatory Syndrome in Children (MIS-C) Compared With Severe Acute COVID-19. JAMA. 2021; 325(11):1074-87. 